PROCEEDINGS OF THE

AMERICAN MATHEMATICAL SOCIETY

Volume 128, Number 12, Pages 3613-3619

S $0002-9939(00) 05511-8$

Article electronically published on June 7, 2000

\title{
ON WEIGHTED POLYNOMIAL APPROXIMATION WITH MONOTONE WEIGHTS
}

\author{
ALEXANDER BORICHEV
}

(Communicated by Albert Baernstein II)

\begin{abstract}
We construct an even weight $W$ monotone on the right half line such that the logarithmic integral of the largest log-convex minorant of $W$ converges and the polynomials are dense in $C(W)$.
\end{abstract}

The problem of weighted polynomial approximation posed by S. Bernstein in 1924 [2] is formulated as follows. Given a weight, that is, a lower semi-continuous function $W: \mathbb{R} \rightarrow[1,+\infty]$ such that $\lim _{|x| \rightarrow \infty} x^{n} / W(x)=0, n \geq 0$, consider the space $C(W)$ consisting of all functions $f$ continuous on $\mathbb{R}$ and such that

$$
\lim _{|x| \rightarrow \infty} f(x) / W(x)=0 .
$$

Identifying elements in $C(W)$ that coincide on the set $\{x \in \mathbb{R}: W(x)=+\infty\}$ and equipping $C(W)$ with the norm $\|f\|=\sup _{x \in \mathbb{R}}|f(x) / W(x)|$, we get a separable normed space. The set $\mathcal{P}$ of all polynomials is a subset of $C(W)$. The weighted polynomial approximation problem is to find out whether the polynomials are dense in $C(W)$.

Note that, if $W, W_{1}$ are weights, $W \leq W_{1}$, and $\operatorname{Clos}_{C(W)} \mathcal{P}=C(W)$, then $\operatorname{Clos}_{C\left(W_{1}\right)} \mathcal{P}=C\left(W_{1}\right)$. Furthermore, if $W: \mathbb{R} \rightarrow[1,+\infty]$ is an arbitrary function, and $W_{0}$ is its lower semicontinuous regularization, $W_{0}(x)=\liminf _{t \rightarrow x} W(t)$, then for every continuous $f, \sup _{x \in \mathbb{R}}|f(x) / W(x)|=\sup _{x \in \mathbb{R}}\left|f(x) / W_{0}(x)\right|$. Thus, in fact, the lower semicontinuity condition on $W$ is not a genuine restriction.

Two (essentially different) solutions to the Bernstein problem were proposed by S. Mergelyan, N. Ahiezer-S. Bernstein and H. Pollard in 1953-1954 and by L. de Branges in 1959 [3]; for more information on the history of the Bernstein problem and related matters see [1], [9, [8], Chapter VI.

Theorem A (S. Mergelyan [9]). The polynomials are dense in $C(W)$ if and only if

$$
\int_{-\infty}^{\infty} \frac{\log W^{*}(x)}{x^{2}+1} d x=+\infty
$$

where $W^{*}(x)=\sup \{|P(x)|: P \in \mathcal{P},|P(t)| \leq(|t|+1) W(t), t \in \mathbb{R}\}$.

Received by the editors February 20, 1999.

2000 Mathematics Subject Classification. Primary 41A10, 46E30.

Key words and phrases. Weighted polynomial approximation, Mergelyan majorant.

(C)2000 American Mathematical Society 
Theorem B (L. de Branges [3). The polynomials are not dense in $C(W)$ if and only if there exists an entire function $F$ of zero exponential type, $F \notin \mathcal{P}$, with simple real zeros $\Lambda_{F}$, such that

$$
\sum_{\lambda \in \Lambda_{F}} \frac{W(\lambda)}{\left|F^{\prime}(\lambda)\right|}<+\infty
$$

The weight $W$ is assumed to be continuous in the original formulation [3]; however, the result holds for lower semicontinuous $W$ as well (see [10]).

The criteria on $W$ in Theorems A and B are not completely explicit. That is why it is rather natural to seek simple necessary (sufficient) conditions for the density of the polynomials. In this direction we mention two results. The first one is contained in a 1937 paper [7] by S. Izumi and T. Kawata. Later on, several authors obtained this result in different forms.

Theorem C. If $W$ is even, $\log W(\exp x)$ is a convex function, and

$$
\int_{-\infty}^{\infty} \frac{\log W(x)}{x^{2}+1} d x=+\infty
$$

then the polynomials are dense in $C(W)$.

Theorem D (T. Hall [5). If

$$
\int_{-\infty}^{\infty} \frac{\log W(x)}{x^{2}+1} d x<+\infty
$$

then the polynomials are not dense in $C(W)$.

Given an even weight $W$ consider its largest (even) log-convex minorant $\widetilde{W}$,

$$
\log \widetilde{W}(\exp x)=\sup \{a x+b: a, b \in \mathbb{R}, a t+b \leq \log W(\exp t), t \in \mathbb{R}\} .
$$

Theorems $\mathrm{C}$ and $\mathrm{D}$ solve the Bernstein problem for even weights $W$ unless

$$
\int_{-\infty}^{\infty} \frac{\log W(x)}{x^{2}+1} d x=+\infty, \quad \int_{-\infty}^{\infty} \frac{\log \widetilde{W}(x)}{x^{2}+1} d x<+\infty .
$$

T. Hall conjectured in [5] that in the case (1), the polynomials are not dense in $C(W)$; L. Carleson produced in [4] a counter-example to this conjecture.

Let us present a version of this example based on (the simple part of) Theorem B. We start with an entire function $B$ of zero exponential type,

$$
B(z)=\prod_{n \geq 0}\left(1-2^{-2 n} z^{2}\right) .
$$

A simple calculation (see [8], p.166) shows that $\left|B^{\prime}\left( \pm 2^{n}\right)\right| \sim C \cdot 2^{-n(n-2)}, n \rightarrow \infty$, and, hence,

$$
\begin{aligned}
& \lim _{n \rightarrow \infty} 2^{-k n}\left|B^{\prime}\left( \pm 2^{n}\right)\right|=\infty, \quad k<\infty, \\
& \lim _{n \rightarrow \infty} 2^{-\varepsilon n} \log \left|B^{\prime}\left( \pm 2^{n}\right)\right|=0, \quad \varepsilon>0 .
\end{aligned}
$$

Furthermore, for every divisor $F$ of $B$ represented by the canonical product of genus zero with zero set $\Lambda_{F}$,

$$
\left|F^{\prime}(\lambda)\right| \leq c(F)\left|B^{\prime}(\lambda)\right|, \quad \lambda \in \Lambda_{F} .
$$

Set $W(x)=\left|B^{\prime}(x)\right|$ if $x= \pm 2^{n}, n \geq 0, W(x)=+\infty$ otherwise. By (2), $W$ is a weight. By (3), $\lim _{|x| \rightarrow \infty}|x|^{-\varepsilon} \log \widetilde{W}(x)=0, \varepsilon>0$, and as a consequence, we get 
the second relation in (1). To verify that the polynomials are dense in $C(W)$, note that for every divisor $F$ of $B$ such that $F \notin \mathcal{P}$ we have by (4),

$$
\sum_{\lambda \in \Lambda_{F}} \frac{W(\lambda)}{\left|F^{\prime}(\lambda)\right|} \geq \frac{1}{c(F)} \sum_{\lambda \in \Lambda_{F}} \frac{\left|B^{\prime}(\lambda)\right|}{\left|B^{\prime}(\lambda)\right|}=+\infty
$$

By Theorem B we obtain that the polynomials are dense, and the first relation in (1) follows from Theorem D.

In this example the weight $W$ behaves rather irregularly; it is a natural question whether the polynomials are dense in $C(W)$ if an even weight $W$ is non-decreasing for $x \geq 0$, and relations (1) hold.

A weight $W$ satisfying these conditions and such that the polynomials are not dense in $C(W)$ is given in [8], Section VII.A.4. (Essentially, this $W$ is equal to $\exp x_{k}$ on $\left(x_{k}, x_{k+1}\right]$ for sufficiently rapidly growing $x_{k}$.)

The aim of our note is to prove the following result.

Theorem 1. There exists an even weight $W$ increasing on the positive half-line and satisfying relations (1) such that the polynomials are dense in $C(W)$.

An elementary argument reduces our problem to that of finding a weight $W_{1}$ increasing on $[0,+\infty)$ and equal to $+\infty$ on $(-\infty, 0)$ such that

$$
\int_{1}^{\infty} \frac{\log \widetilde{W}_{1}(x)}{x^{3 / 2}} d x<+\infty
$$

and the polynomials are dense in $C\left(W_{1}\right)$. Indeed, (5) implies the second relation in (1) for $W(x)=(|x|+1) W_{1}\left(x^{2}\right)$. Suppose that the polynomials are not dense in $C(W)$, that is, $\langle L, \mathcal{P}\rangle=0$ for some $L \in C(W)^{*} \backslash\{0\}$. Define $L_{ \pm} \in C\left(W_{1}\right)^{*}$ by $\left\langle L_{+}, f\right\rangle=\left\langle L, x \rightarrow f\left(x^{2}\right)\right\rangle,\left\langle L_{-}, f\right\rangle=\left\langle L, x \rightarrow x f\left(x^{2}\right)\right\rangle$, and get $\left\langle L_{ \pm}, \mathcal{P}\right\rangle=0$. As the set $C_{0}^{\infty}$ of infinitely differentiable functions with finite support is dense in $C(W)$, and every $g \in C_{0}^{\infty}$ is a sum of $h\left(x^{2}\right)$ and $x h_{1}\left(x^{2}\right)$ for some $h, h_{1} \in C_{0}^{\infty}$, so at least one of $L_{ \pm}$is different from 0 . Thus, the polynomials would not be dense in $C\left(W_{1}\right)$. This contradiction shows that the polynomials are dense in $C(W)$, and by Theorem $\mathrm{D}$, we get the first relation in (1).

Given a function $\varphi$ vanishing on $(-\infty, 0]$ and increasing on $[0,+\infty)$, denote by $\widetilde{\varphi}$ its largest convex minorant, and by $\varphi^{M}$ the Mergelyan majorant, $\varphi^{M}(t)=$ $\sup \{\log |P(\exp t)|: P \in \mathcal{P}, \log |P(\exp x)| \leq \varphi(x), x \in \mathbb{R}\}$.

Theorem 2. There exists a function $\varphi$ vanishing on $(-\infty, 0]$ and increasing on the positive half-line such that $\lim _{t \rightarrow+\infty} \varphi(t) / t=\infty$,

$$
\int_{0}^{\infty} \widetilde{\varphi}(t) e^{-t / 2} d t<+\infty, \quad \int_{0}^{\infty} \varphi^{M}(t) e^{-t / 2} d t=+\infty .
$$

In other words, the functions $\log |P(\exp t)|$ approximate $\varphi$ below much better than linear functions do.

Starting with this $\varphi$, we define $W_{1}(x)=(x+1) \exp \varphi(\log x), x \geq 0, W_{1}(x)=$ $+\infty, x<0$. Then, $W_{1}^{*}(x) \geq \exp \varphi^{M}(\log x), \widetilde{W}_{1}(x) \leq 2 x \exp \widetilde{\varphi}(\log x), x \geq 1$. Therefore, the first of the relations (6) implies inequality (5), and the second one, via Theorem $\mathrm{A}$, implies that the polynomials are dense in $C\left(W_{1}\right)$. Thus, Theorem 1 follows from Theorem 2 . 
Lemma. For every $A \geq 0, \varepsilon>0$ there exist $B<\infty$ and a non-decreasing function $\psi$ vanishing on $(-\infty, A]$ and linear on $[B,+\infty)$ such that $\lim _{t \rightarrow+\infty} \psi(t) / t \geq 1$,

$$
\begin{aligned}
& \int_{A}^{B}\left(R_{L} \psi\right)^{M}(t) e^{-t / 2} d t \geq 1, \\
& \int_{A}^{B} \widetilde{P_{B} \psi}(t) e^{-t / 2} d t \leq \varepsilon \\
& \int_{B}^{\infty} \psi(t) e^{-t / 2} d t \leq \varepsilon
\end{aligned}
$$

where $\left(P_{B} \psi\right)(t)=\varphi(t)$ for $t \leq B,\left(P_{B} \psi\right)(t)=+\infty$ for $t>B,\left(R_{L} \psi\right)(t)=\psi(t)+$ $L \max (t, 1)$, for some constant $L$ to be fixed below.

Proof of Theorem 2. Applying the Lemma with $A_{0}=0, \varepsilon_{0}=1$ we construct $B_{0}$, $\psi_{0}$. Then in an induction process we set $A_{k}=B_{k-1}, \varepsilon_{k}=2^{-k}$ and using the Lemma construct $B_{k}, \psi_{k}, k \geq 1$. Put

$$
\varphi(t)=\sum_{k \geq 0} \psi_{k}(t)+L \max (t, 0), \quad t \in \mathbb{R}
$$

Then

$$
\begin{gathered}
\varphi^{M}(t) \geq\left(R_{L} \psi_{k}\right)^{M}(t)-L, \quad A_{k} \leq t \leq A_{k+1}, \\
\widetilde{\varphi}(t) \leq \sum_{0 \leq j<k} \psi_{j}(t)+\widetilde{P_{B_{k}} \psi_{k}}(t)+L t, \quad A_{k} \leq t \leq A_{k+1}, \\
\liminf _{t \rightarrow \infty} \frac{\varphi(t)}{t} \geq \sum_{0 \leq j<k} \lim _{t \rightarrow \infty} \frac{\psi_{j}(t)}{t} \geq k, \quad k \geq 1 .
\end{gathered}
$$

Therefore,

$$
\lim _{t \rightarrow \infty} \frac{\varphi(t)}{t}=\infty, \quad \int_{0}^{\infty} \widetilde{\varphi}(t) e^{-t / 2} d t<+\infty, \quad \int_{0}^{\infty} \varphi^{M}(t) e^{-t / 2} d t=+\infty .
$$

Proof of the Lemma. We are going to find numbers $D, G, B, 4 A \leq 2 D \leq B / 2 \leq$ $G \leq B$, a non-decreasing function $\psi$ that vanishes on $(-\infty, A]$, is a constant on $(A, G]$, and is linear on $[B,+\infty)$, and a function

$$
h_{\nu}(x)=\int \log \left|e^{x-t}-1\right| d \nu(t),
$$

where $\nu$ is a finite positive measure with support on $[A, B], d \nu / d t \leq S e^{t / 2}$ for some constant $S$ that does not depend on $A$ and $\varepsilon$, such that

(i) $h_{\nu}(x) \leq \psi(x), \quad x \geq 0$.

(ii) $h_{\nu}(x) \geq e^{x / 2+1}, \quad D \leq x \leq D+1$.

(iii) $\psi(G) \leq \varepsilon(G-A) / 2$.

(iv) $\int_{G}^{\infty} \psi(x) e^{-x / 2} d x \leq \varepsilon / 2$.

(v) $\psi(x) \geq x, \quad x \geq B$.

A standard argument permits us to produce a measure $\mu$ equal to a finite sum of positive integer point masses, $\operatorname{supp} \mu \subset[A, B]$, such that the function $h_{\mu}(x)=$ 
$\int \log \left|e^{x-t}-1\right| d \mu(t)$ approximates $h_{\nu}$ :

$$
\begin{array}{rlrl}
h_{\mu}(x)-h_{\nu}(x) & \leq L \max (x, 1), & & x \geq 0, \\
h_{\nu}(x)-h_{\mu}(x) \leq L \max (x, 1), & & x \in[0,+\infty) \backslash E,
\end{array}
$$

where $L$ depends only on $S,|E| \leq 1 / 2$. The function $P(x)=\exp h_{\mu}(\log x)$ is a polynomial, $|P(x)| \leq 1,0 \leq x \leq 1$, and the assertions of the lemma follow from estimates (i)-(v) if $D \geq D_{0}(L), \exp \left(D_{0}(L) / 2\right)=10 L\left(D_{0}(L)+1\right)$.

One way to get $\mu$ is to define $\mu(-\infty, x]$ to be equal to the integral part of $\nu(-\infty, x], x \in \mathbb{R}$, and to use the estimates for the Kjellberg-Kennedy-Katifi approximation method given in [6], Section 10.5.3. Alternatively, we may use the Yulmuhametov approximation technique (11, Theorem 2). As a result, the function $Y=\left(h_{\mu}-h_{\nu}\right) \circ \log$ extended harmonically to $\mathbb{C} \backslash[A, B]$ satisfies the estimate $|Y(z)| \leq K \log (|z|+1), z \in \mathbb{C} \backslash \mathcal{E}$, where $\mathcal{E}$ is an exceptional set consisting of disjoint discs with finite sum of radii. This gives us (8). Since $d \nu / d t \leq S e^{t / 2}$, the function $Y(z)+S|\operatorname{Im} z|$ is subharmonic in the plane, and, applying the maximum principle to this function, we get (7).

In our construction we use some properties of a function $r \in C(\mathbb{R}) \cap C^{1}(\mathbb{R} \backslash\{0\})$,

$$
r(x)=\int_{x}^{\infty} \log \left|e^{t}-1\right| e^{-t / 2} d t, \quad x \in \mathbb{R} .
$$

The function $r$ increases on $(-\infty, \log 2]$ and decreases on $[\log 2,+\infty), r^{\prime} \in L^{1}(\mathbb{R})$. An elementary calculation using integration by parts shows that

$$
\frac{1}{2} \int_{-\infty}^{\infty} \log \left|e^{t}-1\right| e^{-t / 2} d t=0,
$$

and, as a consequence, we have $\lim _{x \rightarrow \pm \infty} r(x)=0$. Therefore,

$$
r(x)=-\int_{-\infty}^{x} \log \left|e^{t}-1\right| e^{-t / 2} d t, \quad x \in \mathbb{R},
$$

$r$ is strictly positive on $\mathbb{R}$, and for some $Q>1$,

$$
\begin{aligned}
\frac{1}{Q} e^{x / 2} & \leq r(x) \leq Q e^{x / 2}, \quad x \leq 0, \\
\frac{x+1}{Q} e^{-x / 2} & \leq r(x) \leq Q(x+1) e^{-x / 2}, \quad x \geq 0 .
\end{aligned}
$$

For some $D, B$ to be fixed below, $8 A \leq 4 D \leq B$, put

$$
d \nu(t)=e^{t / 2}\left[10 Q \chi_{[A, D]}(t)+\frac{\varepsilon}{20 Q} \chi_{[2 D, B]}(t)\right] d t .
$$

Then

$$
\begin{gathered}
h_{\nu}(x)=e^{x / 2}\left[10 Q \int_{x-D}^{x-A} \log \left|e^{t}-1\right| e^{-t / 2} d t+\frac{\varepsilon}{20 Q} \int_{x-B}^{x-2 D} \log \left|e^{t}-1\right| e^{-t / 2} d t\right] \\
=-e^{x / 2}\left[10 Q \int_{x-D}^{x-A} r^{\prime}(t) d t+\frac{\varepsilon}{20 Q} \int_{x-B}^{x-2 D} r^{\prime}(t) d t\right] \\
=e^{x / 2}\left[-10 \operatorname{Qr}(x-A)+10 \operatorname{Qr}(x-D)-\frac{\varepsilon}{20 Q} r(x-2 D)+\frac{\varepsilon}{20 Q} r(x-B)\right] .
\end{gathered}
$$


Using the properties of $r$ formulated above we get

$$
\begin{aligned}
& h_{\nu}(x) \leq 0, \quad 0 \leq x \leq A, \\
& h_{\nu}(x) \geq e^{x / 2+1}, \quad D \leq x \leq D+1, \quad(5 / 3) D \leq x \leq B / 2, \\
& h_{\nu}(x) \leq 0, \quad B / 2 \leq x \leq B, \\
& h_{\nu}(x) \leq \frac{\varepsilon}{20 Q} r(x-B) e^{x / 2} \leq \begin{cases}\frac{\varepsilon}{20} e^{x-B / 2}, \quad x \geq B, \\
\frac{\varepsilon}{20}(x-B+1) e^{B / 2}, \quad x\end{cases}
\end{aligned}
$$

if $D-2 A \geq c(Q), B-4 D \geq c(Q)$. Put

$$
H=10 Q e^{x / 2} \max _{0 \leq x \leq(5 / 3) D}(r(x-D)-r(x-A)) \geq \max _{0 \leq x \leq(5 / 3) D} h_{\nu}(x) .
$$

From now on we fix $B$ and $D$ such that $D-2 A \geq c(Q), B-4 D \geq c(Q), B \geq 10 H / \varepsilon$, $\varepsilon e^{B / 2}>20 B, D \geq D_{0}(L)$. Define $G$ by the equality $(\varepsilon / 20) e^{G-B / 2}=H$. Then $B / 2 \leq G<B$. Put

$$
\psi(x)=\left\{\begin{array}{l}
0, \quad x \leq A, \\
H, \quad A<x \leq G, \\
\frac{\varepsilon}{20} e^{x-B / 2}, \quad G<x \leq B, \\
\frac{\varepsilon}{20}(x-B+1) e^{B / 2}, \quad x>B .
\end{array}\right.
$$

We have already verified inequalities (i) and (ii). Inequality (v) follows from our assumption $\varepsilon e^{B / 2}>20 B$. Inequality (iii) is proved as follows:

$$
\psi(G)=H \leq \frac{\varepsilon}{10} B \leq \frac{\varepsilon}{5} G \leq \frac{\varepsilon}{2}(G-A) .
$$

It remains to note that

$$
\frac{\varepsilon}{20}\left[\int_{G}^{B} e^{(x-B) / 2} d x+\int_{B}^{\infty}(x-B+1) e^{(B-x) / 2} d x\right] \leq \frac{\varepsilon}{2} .
$$

The lemma is proved.

Remark. A simple modification of our argument permits us to produce continuous $W$ satisfying the conditions of Theorem 1 .

The author thanks Mikhail Sodin for numerous helpful discussions.

\section{REFERENCES}

1. N. Ahiezer, On the weighted approximation of continuous functions by polynomials on the entire number axis, Uspekhi Mat. Nauk 11 (1956), 3-43; English translation in Amer. Math. Soc. Translations, Ser. 2, vol. 22, 1962, pp. 95-137. MR 18:802f

2. S. Bernstein, Le problème de l'approximation des fonctions continues sur tout l'axe réel et l'une de ses applications, Bull. Soc. Math. France (52) (1924), 399-410.

3. L. de Branges, The Bernstein problem, Proc. Amer. Math. Soc. 10 (1959), 825-832. MR 22:4907

4. L. Carleson, On Bernstein's approximation problem, Proc. Amer. Math. Soc. 2 (1951), 953961. MR 13:632d

5. T. Hall, Sur l'approximation polynômiale des fonctions continues d'une variable réelle, Neuvième Congrès des Mathématiciens Scandinaves (1938), Helsingfors, 1939, 367-369.

6. W. K. Hayman, Subharmonic functions, volume II, Academic Press, 1989. MR 91f:31001

7. S. Izumi, T. Kawata, Quasi-analytic class and closure of $\left\{t^{n}\right\}$ in the interval $(-\infty, \infty)$, Tôhoku Math. J. (43) (1937), 267-273.

8. P. Koosis, The logarithmic integral, vol. I, Cambridge University Press, Cambridge, 1988, 606 pp. CMP 99:07 
9. S. Mergelyan, Weighted approximation by polynomials, Uspekhi Mat. Nauk 11 (1956), 107152; English translation in Amer. Math. Soc. Translations, Ser. 2 , vol. 10, 1958, pp. 59-106. MR 20:1146

10. M. Sodin and P. Yuditskii, Another approach to de Branges' theorem on weighted polynomial approximation, in: Proceedings of the Ashkelon Workshop on Complex Function Theory (May 1996), L. Zalcman, ed., Israel Mathematical Conferences Proceedings vol. 11, Amer. Math. Soc., Providence RI, 1997, pp. 221-227. MR 99c:41014

11. R. Yulmuhametov, Approximation of subharmonic functions, Anal. Math. 11 (1985), 257-282 [in Russian]. MR 88a:31002

Laboratoire de Mathématiques Pures de Bordeaux, UpReSA 5467 CNRS, Université Bordeaux I, 351, cours de la Libération, 33405 Talence, France

E-mail address: borichev@math.u-bordeaux.fr 